\title{
Theta Phase Precession in Rat Ventral Striatum Links Place and Reward Information
}

\author{
Matthijs A. A. van der Meer ${ }^{1,2}$ and A. David Redish ${ }^{1}$ \\ ${ }^{1}$ Department of Neuroscience, University of Minnesota, Minneapolis, Minnesota 55455, and ${ }^{2}$ Department of Biology and Centre for Theoretical \\ Neuroscience, University of Waterloo, Waterloo, Ontario, Canada N2L 3G1
}

A functional interaction between the hippocampal formation and the ventral striatum is thought to contribute to the learning and expression of associations between places and rewards. However, the mechanism of how such associations may be learned and used is currently unknown. We recorded neural ensembles and local field potentials from the ventral striatum and CA1 simultaneously as rats ran a modified T-maze. Theta-modulated cells in ventral striatum almost invariably showed firing phase precession relative to the hippocampal theta rhythm. Across the population of ventral striatal cells, phase precession was preferentially associated with an anticipatory ramping of activity up to the reward sites. In contrast, CA1 population activity and phase precession were distributed more uniformly. Ventral striatal phase precession was stronger to hippocampal than ventral striatal theta and was accompanied by increased theta coherence with hippocampus, suggesting that this effect is hippocampally derived. These results suggest that the firing phase of ventral striatal neurons contains motivationally relevant information and that phase precession serves to bind hippocampal place representations to ventral striatal representations of reward.

\section{Introduction}

Two domains in which the hippocampal formation plays a critical role, rapid encoding and retrieval of experience, and flexible spatial navigation, are thought to share fundamental information processing elements (O'Keefe and Nadel, 1978; Eichenbaum et al., 1999; Redish, 1999). In particular, a sequence of "place cells," activated as rodents traverse a path, can be thought of as a memory episode, potentially subject to encoding, consolidation, and retrieval processes. A key mechanism proposed to support the rapid encoding (storage) process is the precise temporal relationship of hippocampal spikes to the ongoing theta rhythm (7-10 $\mathrm{Hz}$ ). O'Keefe and Recce (1993) found that the theta firing phase of a place cell advanced as the animal passed through its field, a phenomenon known as "phase precession." As a result, sequences of place cell spiking are effectively compressed in time, thought to facilitate plasticity mechanisms that may depend on precise spike timing (Skaggs et al., 1996; Bi and Poo, 1998; Lisman, 2005; Dragoi and Buzsáki, 2006; Robbe and Buzsáki, 2009).

Typically, studies of phase precession have been restricted to spatial or temporal sequences in the hippocampal formation (Skaggs et al., 1996; Mehta et al., 1997, 2000; Harris et al., 2002; Maurer et al., 2006; Hafting et al., 2008; Pastalkova et al., 2008; Harvey et al., 2009; Schmidt et al., 2009) (but see Jones

Received Sept. 15, 2010; revised 0ct. 21, 2010; accepted Dec. 17, 2010.

This work was supported by National Institutes of Health Grant MH080318 (A.D.R.). We thank Anoopum Gupta, Carien Lansink, and Sebastian Royer for discussion, Tobias Bast for discussion and helpful comments on a previous draft of this manuscript, and Chris Boldt and Kelsey Seeland for technical assistance.

Correspondence should be addressed to Matthijs van der Meer, Department of Biology, University of Waterloo, 200 University Avenue West, Waterlo0, 0N, Canada, N2L 3G1. E-mail: mvdm@uwaterloo.ca.

DOI:10.1523/JNEUROSCI.4869-10.2011

Copyright $\odot 2011$ the authors $\quad 0270-6474 / 11 / 312843-12 \$ 15.00 / 0$ and Wilson 2005a). However, the hippocampal formation sends projections to several structures, including prefrontal cortex (Swanson, 1981) and the ventral striatum (VS) (Groenewegen et al., 1987). Both structures exhibit coherent theta rhythmicity (Tabuchi et al., 2000; Martin, 2001; Jones and Wilson, 2005a; Siapas et al., 2005; Adhikari et al., 2010), theta phase locking (Jones and Wilson, 2005b; Benchenane et al., 2010; Hyman et al., 2010), and coordinated "replay" with the hippocampus (HC) (Lansink et al., 2009; Peyrache et al., 2009; Benchenane et al., 2010), suggesting a relationship to hippocampal temporal coding schemes. In general, the hippocampus-ventral striatal projection has long been implicated in the modulation of motivated behavior (Mogenson et al., 1980; Pennartz et al., 1994; Bast and Feldon, 2003; van der Meer and Redish, 2010). In particular, a recent study disconnecting the hippocampus and ventral striatum (nucleus accumbens shell) found impairments in a conditioned place preference task, suggesting that this projection supports the learning of place-reward associations (Ito et al., 2008).

Thus, a functional projection from the hippocampal formation to the ventral striatum may support the learning and expression of place-reward relationships. Ventral striatum has been shown previously to contain anticipatory reward-related firing on spatial tasks (Carelli and Deadwyler, 1994; Lavoie and Mizumori, 1994; Miyazaki et al., 1998; Daw, 2003; Khamassi et al., 2008; van der Meer and Redish, 2009a). Such anticipatory "ramping" neurons are a candidate for linking place and reward information. Here, we report that, on the multiple-T task (van der Meer et al., 2010a), ventral striatal "ramp" neurons phase precessed relative to the hippocampal theta rhythm, suggesting a temporal coding mechanism associating place and reward information. 


\section{Materials and Methods}

\section{Subjects}

Five male Brown Norway-Fisher 344 hybrid rats (Harlan), aged 8-11 months at the start of behavioral training, were trained to run the multiple-T task, as described previously (van der Meer and Redish, $2009 \mathrm{~b}$ ). Rats were food deprived to no less than $80 \%$ of their free-feeding body weight during behavioral training; water was available ad libitum in the home cage at all times. Animals were housed individually and maintained on a $12 \mathrm{~h}$ light/dark cycle, with the lights on during the day, when training and recording sessions took place. All procedures were conducted in accordance with National Institutes of Health guidelines for animal care and approved by the Institutional Animal Care and Use Committee at the University of Minnesota. Care was taken to minimize the number of animals used in these experiments and to minimize suffering.

\section{Multiple-T task and training}

We used a variant of the multiple-T task, consisting of a continuous elevated track with a sequence of T-choices (T1-T3) and a final choice point (T4) between two return arms, "left" and "right" (SchmitzerTorbert and Redish, 2004). Both return arms were equipped with two reward delivery sites; as in the study by Gupta et al. (2010), the first reward site on the left delivered banana-flavored pellets (5TUL-banana; TestDiet) and on the right, fruit-flavored pellets (5TUL-fruit). On both left and right return arms, the second reward site delivered white unflavored pellets (5TUL). Rats ran $40 \mathrm{~min}$ sessions during both training and recording sessions, one session per day per subject. The configuration of turns T1-T3 was changed daily, for instance from "LLL" to "RLR," etc. For recording sessions, the location of the final choice point T4 was always in the same physical location, equidistant from the two return rails.

Training on the task was performed in two phases. During phase 1, rats were trained to run laps on one side of the maze, whereas the other side was blocked. After running at least 40 laps on 2 consecutive days, phase 2 of training began. Blocks were removed, and, on a given day, rats had to turn left at the final choice point T4 (L, corresponding to left laps), turn right ( $R$, right laps), or alternate left and right laps (A) to receive reward (at both sites on the correct side). After an incorrect (unrewarded) choice, rats were prevented from turning around. After consistently getting $80 \%$ of the laps correct on all three reward schedules, rats were surgically implanted with recording electrodes. After recovery from surgery, phase 2 of training resumed until the rats were running proficiently. At this point, recording began. After recording a number of sessions, a $6 \mathrm{~d}$ sequence of "switch" sessions began, in which the reward schedule changed approximately midway through the session (Gupta et al., 2010). There were six recording sessions to allow for all possible pairings of the three tasks ( $\mathrm{L}-\mathrm{R}, \mathrm{R}-\mathrm{L}, \mathrm{L}-\mathrm{A}, \mathrm{A}-\mathrm{L}, \mathrm{R}-\mathrm{A}, \mathrm{A}-\mathrm{R})$. The order that each rat experienced the six potential pairings was randomized across animals. Because no differences were found between switch and non-switch recording sessions, both are included in our analyses here.

\section{Surgery and data collection}

After pretraining to proficiency, rats were implanted with a doublebundle 12-tetrode, two-reference microdrive directed toward CA1 and ventral striatum (CA1 targets: $3.8 \mathrm{~mm}$ posterior and $2.5 \mathrm{~mm}$ right-lateral from bregma, eight tetrodes and one reference; VS targets: $1.6-2.0 \mathrm{~mm}$ anterior to bregma and $2.0 \mathrm{~mm}$ right-lateral, four tetrodes and one reference). Tetrodes were constructed from 0.0127-mm-diameter wire insulated with polyamide (Kanthal Precision Wire) and gold plated to impedances in the $250-300 \mathrm{k} \Omega$ range (measured at $1 \mathrm{kHz}$; BAK Electronics IMP-1). Reference electrodes consisted of tetrodes with all four channels connected at the interface board level (before the head-stage pre-amps), gold plated to $200-250 \mathrm{k} \Omega$ impedance. Details of the surgical procedure were as presented previously (Johnson and Redish, 2007). After the completion of experiments, the location of each tetrode was marked by passing a small amount of current through each tetrode (10 $\mu \mathrm{A}$ for $5-10 \mathrm{~s})$ for subsequent histological localization.

Neural activity was recorded using a 64-channel Cheetah recording system (Neuralynx), allowing for the simultaneous recording of unit and
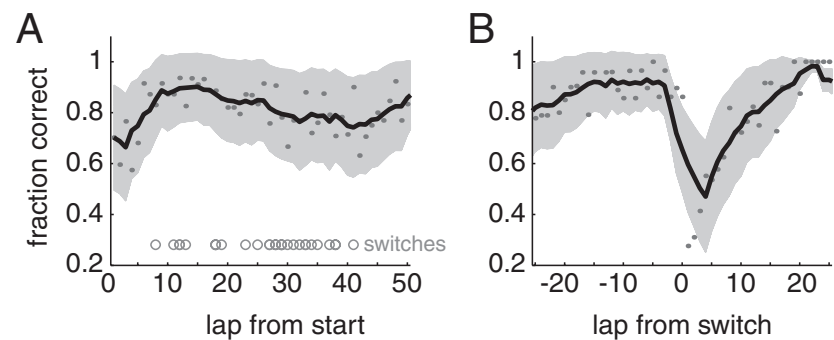

Figure 1. Behavioral performance. Rats rapidly learned to choose the correct (rewarded) side both from session start $(\boldsymbol{A})$ and after a contingency switch approximately midway through switch sessions $(\boldsymbol{B})$. Gray dots indicate the proportion of correct choices at each lap; the black line is the five-lap running average, with the shaded area SEM over subjects. In the left, laps at which a switch occurred are indicated by the gray circles. Left, right, and alternate sessions were averaged together.

local field potential (LFP) activity, passed through different amplifiers. LFPs were continuously sampled at $1990.4 \mathrm{~Hz}$, amplified 500 or 1000 times, bandpass filtered between 1 and $475 \mathrm{~Hz}$ (hardware filters, Cheetah recording system; Neuralynx), and recorded to disk, with at least one LFP recorded from each tetrode. When multiple LFPs were recorded from the same tetrode, only one was included for analysis. The CA1 bundle reference electrode was placed in the hippocampal fissure, and the ventral striatum reference was placed in or near the corpus callosum. Spike data were referenced against the HC reference and LFP data against the ventral striatal reference. Spiking activity was recorded to disk at $32 \mathrm{kHz}$ when an experimenter-set voltage threshold was met. Putative single neurons ("units") were isolated using MClust 3.5 (A. D. Redish, http://redishlab. neuroscience.umn.edu/MClust/MClust.html) after automatic preclustering with KlustaKwik 1.7 (K. Harris, http://klustakwik.sourceforge.net/), using Condor distributed computing (http://www.cs.wisc.edu/condor/).

\section{Data analysis}

Behavioral analyses. To allow comparison across different maze configurations, animal position data were linearized and warped to two experimenter-drawn idealized paths (Schmitzer-Torbert and Redish, 2004), one path for left laps and one for right laps. Because no systematic differences between left and right laps were found (consistent with the data in the study by Gupta et al., 2010), data from both paths were subsequently averaged, weighted by the number of laps for left and right. Note that, for recording sessions, the final choice point (T4) was always in the same physical location, so no stretching or compression of space was required to compare firing patterns across different sessions. All data in which the rat deviated $>10 \mathrm{~cm}$ from the relevant path or when the animal's head movement (as estimated from light-emitting diodes mounted on the recording head stage) was $<3 \mathrm{~cm} / \mathrm{s}$ were excluded from analysis.

Local field potential preprocessing. Before all analysis, direct current offsets and slowly changing components were removed from all LFP recordings by applying the locdetrend function in the Chronux 2.00 toolbox (http://www.chronux.org) (Mitra and Bokil 2008), which subtracts the linear regression line fit within a $1 \mathrm{~s}$ moving window.

Average power spectral density estimation. Average power spectral densities (PSDs) were constructed for each 40 min recording session by first decimating the raw LFP by a factor 3. A Welch spectral estimator (Percival and Walden, 1993, section 6.17) was then applied to obtain the PSD (MATLAB Signal Processing Toolbox, spectrum.welch; this effectively implements Equation 3 below, using a single Hamming window taper and $50 \%$ overlapping $1.5 \mathrm{~s}$ time windows).

Theta phase estimation. To obtain a theta phase angle for each spike, LFPs were first bandpass filtered (fourth-order Chebyshev, $r=0.5$, MATLAB filter and filtfilt routines; $6-10 \mathrm{~Hz}$ ) before a Hilbert transform was applied to obtain the instantaneous phase angle.

Local field potential coherency estimation. To estimate to what extent the theta rhythm was coherent between hippocampal and ventral striatal recording sites, coherograms were computed for two LFP pairs per session: the hippocampal fissure electrode and a ventral striatal electrode, 


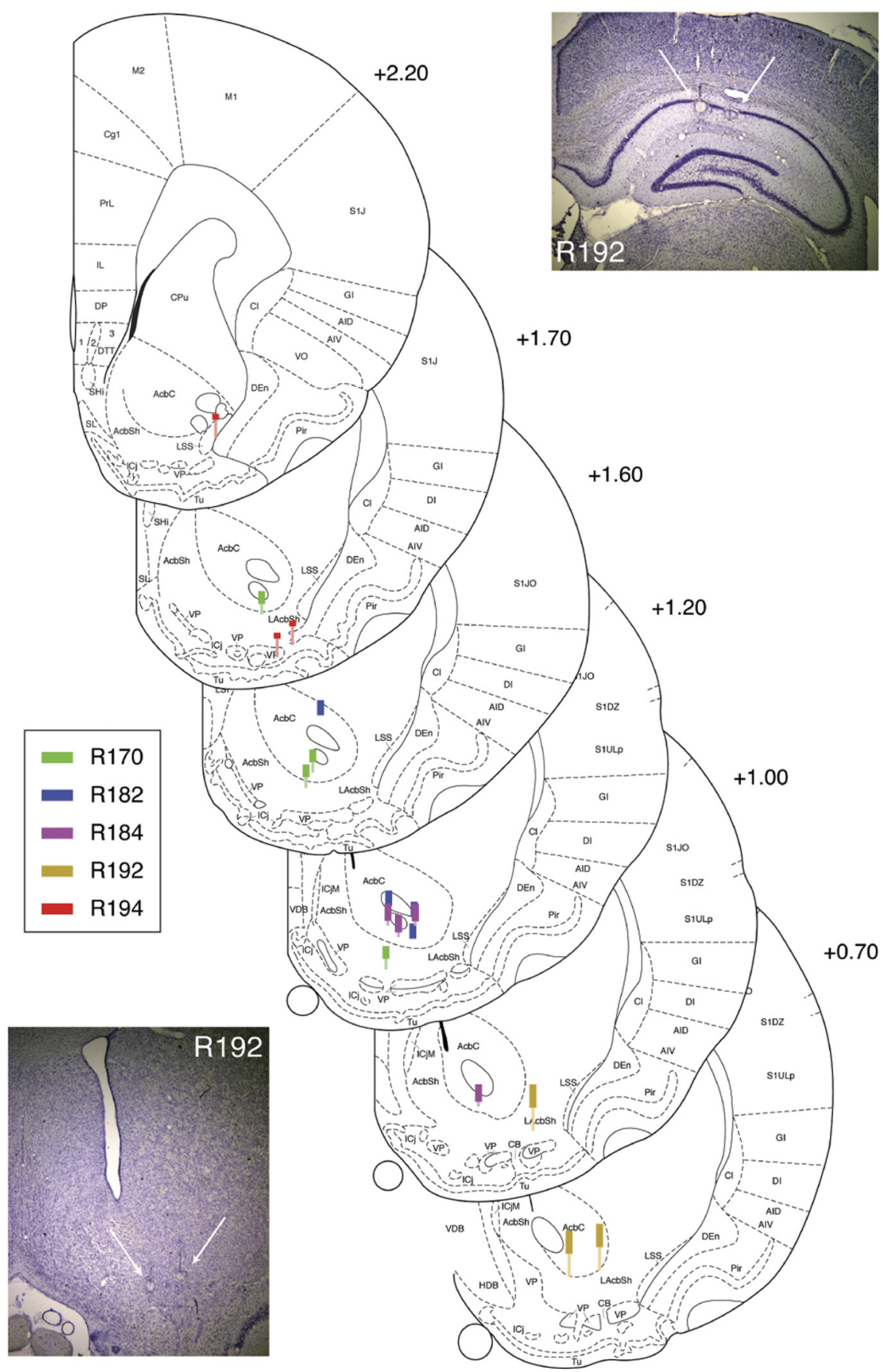

Figure 2. Recording locations. Thick lines indicate estimated recording areas based on distance from final recording locations identified histologically (bottom of thin lines). Colors correspond to different subjects. All subjects were implanted in the right hemisphere; coordinates are relative to bregma. Example cresyl-violet-stained brain sections shown from one subject (R192); final electrode locations can be seen from the small round electrolysis marks (white arrows). Ventral striatal recording locations were all estimated to be from the nucleus accumbens core and shell; although some electrodes passed through the anterior commissure, this tended to be clearly apparent as a quiet area for recording, and electrodes were rapidly advanced until neuronal activity was reacquired. Figure based on Paxinos and Watson (1998).

and a hippocampal layer electrode and the same ventral striatal electrode. Coherograms estimate the coherency $C$ between the two LFP power spectra $X$ and $Y$, for each frequency $f$ as follows:

$$
C_{X Y}(f)=\frac{S_{X Y}(f)}{\sqrt{S_{X X}(f) S_{Y Y}(f)}}
$$

with

$$
S_{X Y}(f)=\frac{1}{K} \sum_{k=1}^{K} x_{k}(f) y_{k}(f)
$$

where $x_{k}$ and $y_{k}$ are the two LFP spectra:

$$
x_{k}(f)=\sum_{t=1}^{T} w_{t}(k) x_{t} e^{-2 \pi i f t} .
$$

To obtain fine-timescale estimates of coherency, we used the Chronux cohgramc function, which implements a multi-taper estimate (the index $k$, above) of the spectra (Percival and Walden, 1993; Mitra and Bokil, 2008), with the following parameters: window size, $0.5 \mathrm{~s}$; time step, $100 \mathrm{~ms}$; five tapers; $6 \mathrm{~Hz}$ bandwidth. To obtain a theta band coherence time series, measuring the constancy in phase between the two sites, we averaged the real (magnitude) component of the coherency across frequencies in the 6-10 Hz band; similar results were obtained when averaging in the $7-9 \mathrm{~Hz}$ band.

Cell type categorization. As described previously, ventral striatal units were assigned to one of three cell type categories: phasically firing neuron (putative medium spiny neuron), high-firing neuron (putative fast-spiking interneuron), or tonically firing neuron, based on spiking statistics (Schmitzer-Torbert and Redish, 2008; van der Meer et al., 2010a). Hippocampal units were separated into putative pyramidal neurons and interneurons based on average firing rate (interneurons, $>2 \mathrm{~Hz}$ ) (Ranck 1973; Csicsvari et al., 1999). For all analyses except the population spike train autocorrelograms in Figure 11, only putative medium spiny neurons in ventral striatum and putative pyramidal neurons in hippocampus were included.

Theta index estimation. To quantify the degree and frequency of theta modulation in single cells, we used the method used by Royer et al. (2010). First we computed the autocorrelogram of the cell, in $10 \mathrm{~ms}$ bins from -500 to $+500 \mathrm{~ms}$, normalized it to the maximum value between 100 and $150 \mathrm{~ms}$ (corresponding to theta modulation), and clipped all values above 1. Then we fit the following function:

$$
\begin{aligned}
y(t)=[a \cdot(\sin (\omega t)+1)+b] \cdot & e^{-|t| / \tau_{1}} \\
& +c \cdot e^{-t^{2} / \tau_{2}^{2}},
\end{aligned}
$$

where $t$ is the autocorrelogram time lag, and $a-c, \omega$, and $\tau_{1-2}$ were fit using the fminsearch optimization function in MATLAB. A measure of theta modulation strength, the "theta index," was defined as $a / b$, which intuitively corresponds to the ratio of the sine fit relative to the baseline in the autocorrelogram. We elected to use this measure rather than spectral methods because of the direct estimate of the theta frequency $\omega$ it provides, which in the case of a spectral estimator needs to be obtained indirectly. For best-fitting performance, we restricted possible values for $\omega$ to $[6,12]$, for $a$ and $b$ to non-negative values, for $c$ to $[0,0.2]$, and for $\tau_{2}$ to $[0,0.05]$. Accuracy of this fitting procedure was further improved by only including spike data from when animals were active (moving their heads at $>3 \mathrm{~cm} / \mathrm{s}$ ) as described above (see Behavioral analysis) and by only fitting cells with at least 100 spikes available. Examples of the fit output can be seen in Figures 3 and 4 . 
Phase precession detection. We defined a cell as phase precessing when there was a significant $(p<0.05)$ linear-circular correlation (Zar, 1999) between linearized location on the track and firing phase.

Phase-rate analysis. To investigate the relationship between instantaneous firing rate and theta firing phase of ventral striatal cells, an average phase for each spatial bin $(n=100$ bins along the linearized track) was obtained for each spike train. Following Mehta et al. (2002), a scatter plot of average firing phase against average firing rate was constructed in which each point corresponds to one spatial bin for one putative neuron. For clarity, only bins with circular confidence intervals $(95 \%)$ smaller than $\pi / 4$ were included.

\section{Results}

Rats $(n=5)$ ran a variation of the multiple- $\mathrm{T}$ task in which a changing sequence of "low-cost" turns (T1-T3) was followed by a choice point (T4) between the left and right return rails (SchmitzerTorbert and Redish, 2004; van der Meer and Redish, 2009a). Both arms contained two food reward sites but, on any given trial, only one side was rewarded: sessions could have left, right, or alternate reward schedules (Gupta et al., 2010). During training (before surgery), only one reward schedule was in effect for a session. After surgery, during recording, we ran both single reward schedule sessions, as well as switch sessions in which the reward schedule was changed approximately midway through the session, unbeknownst to the rat. Each rat experienced all six possible switches ( $\mathrm{L}-\mathrm{R}, \mathrm{L}-\mathrm{A}, \mathrm{R}-\mathrm{L}, \mathrm{R}-\mathrm{A}, \mathrm{A}-\mathrm{L}$, $\mathrm{A}-\mathrm{R}$ ) in pseudorandom order. As in the study by Gupta et al. (2010), rats started out at chance performance at the beginning of the session and rapidly learned to choose correctly (Fig. $1 A$ ). After the switch, subjects dropped to the performance level expected from persisting with their previous strategy but recovered to near perfect performance within 5-10 trials (Fig. $1 B$ ).

Subjects were surgically implanted with dual-bundle electrode arrays, with four tetrodes (plus a reference) aimed at the ventral striatum and eight tetrodes (plus an additional electrode with the four wires connected for field potential recording) aimed at the dorsal CA1 subfield of the hippocampus. Subsequent histological localization indicated all of the recording sites to be at the intended targets (Fig. 2). LFPs were recorded from the single electrode placed in the hippocampal fissure, where theta power tends to be largest. In line with previous results, hippocampal units tended to form well-defined "place fields," whereas ventral striatal units showed a wider range of firing patterns, including more diffuse spatial fields, sharp reward-related responses, and gradual ramping up to the reward sites. To study spike timing relationships with the ongoing theta rhythm, we assigned each spike a phase value obtained from the theta-band-
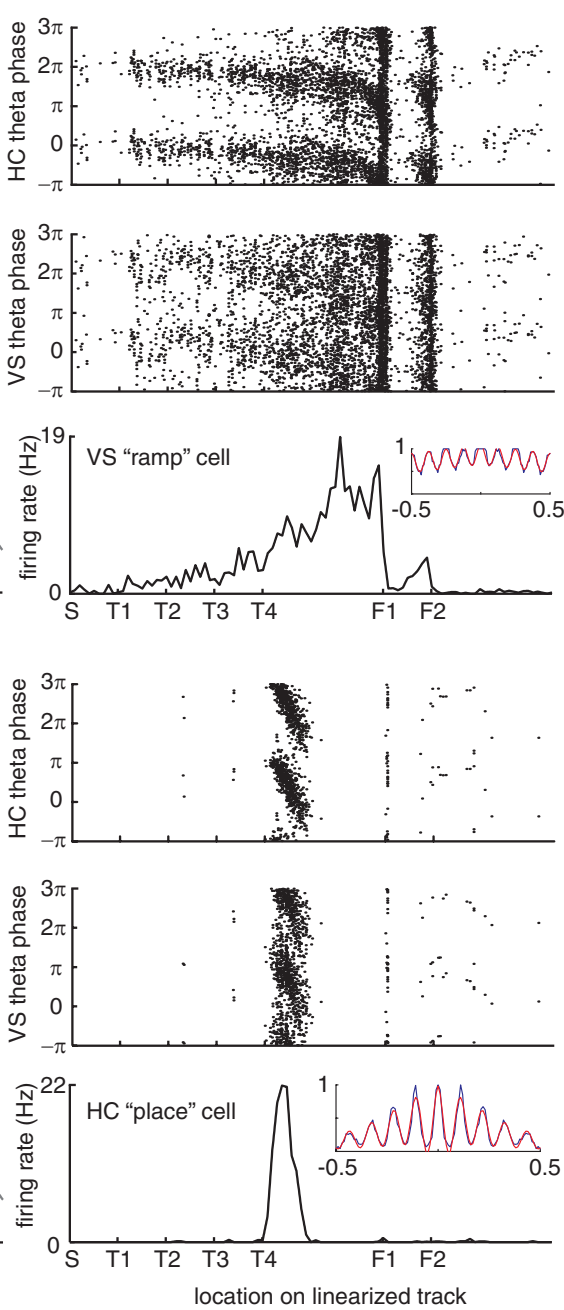

Figure 3. Examples of theta phase precession in a simultaneously recorded ramp cell in ventral striatum $(\boldsymbol{A})$ and a place cell in CA1 $(B)$. Top left, Each spike is plotted at the location on the track where it occurred and color coded for theta phase. Notice how as the animal progresses through the turn sequence (T1-T4) and approaches the first reward site (F1) spike theta phase precesses. precession toward the reward sites. For comparison, the same plot is shown with phase calculated relative to ventral striatal, rather

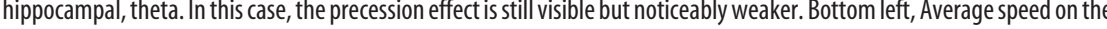
arage firing rate on the linearized track; notice the large ramp up to the first reward site (F1) and a smaller ramp up to F2. Inset shows the autocorrelogram of this unit (blue line) and its best fit (red line) used for calculating a theta modulation index (see Materials and Methods). Taken from session R170-2009-08-12, tetrode 3 cell $2(\boldsymbol{A})$ and tetrode 9 cell $8(\boldsymbol{B})$.

filtered fissure LFP $(6-10 \mathrm{~Hz})$, restricting analyses to epochs when the animal was active (speed $>3 \mathrm{~cm} / \mathrm{s}$ ). As in previous reports, hippocampal cells showed clear phase precession as animals passed through their place field (O'Keefe and Recce 1993; Skaggs et al., 1996; Mehta et al., 2002; Dragoi and Buzsáki 2006) (Figs. 3, 4B).

\section{Theta phase precession in ventral striatal ramp cells}

We found striking examples of phase precession in ventral striatal ramp neurons (Figs. 3, 4A). Typically, as reported previously (Schultz et al., 1992; Carelli and Deadwyler, 1994; Miyazaki et al., 1998; Khamassi et al., 2008), these ramp neurons gradually increased their firing rate as rats approached the first reward site (F1), abruptly switching off before increasing up to the second reward site (F2), as can be seen from the tuning curve in the bottom right panels in Figures 3 and 4 . This firing rate profile was not clearly related to running speed (Figs. 3, 4, gray line in bottom 
A


B
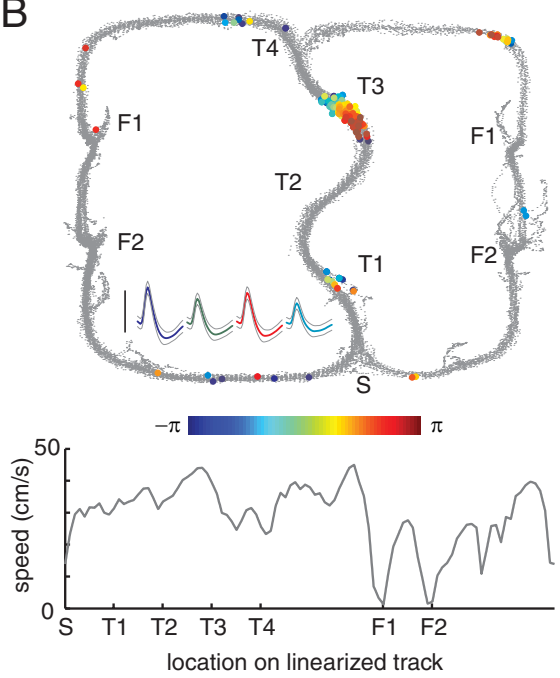
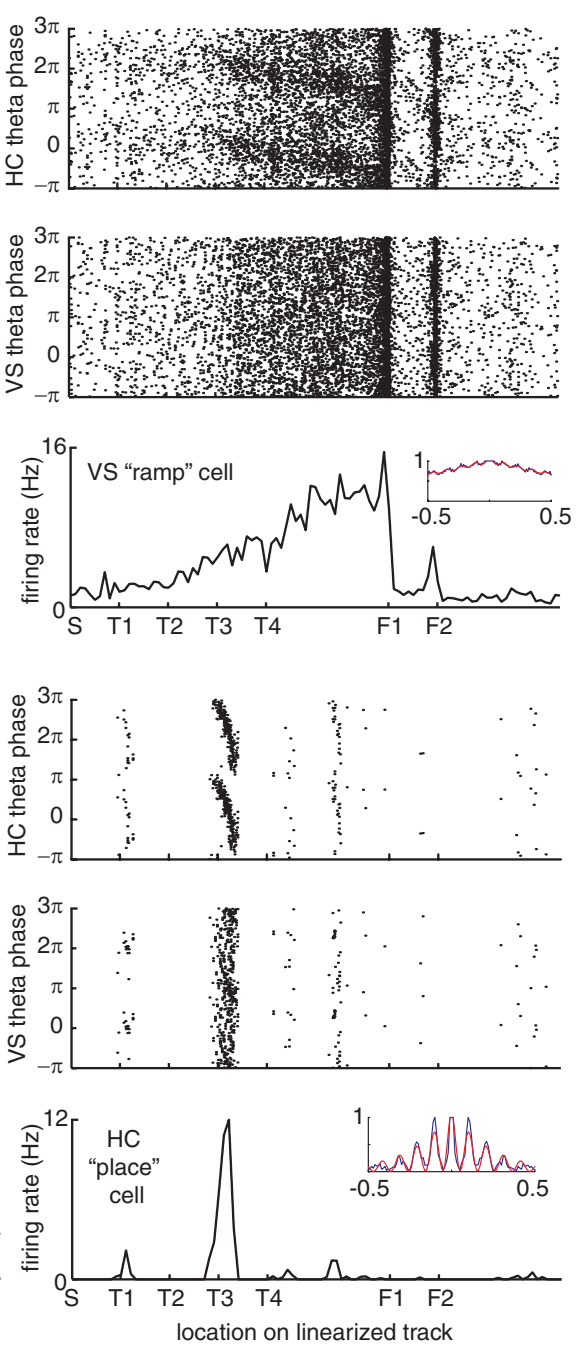

Figure 4. Examples of theta phase precession in a simultaneously recorded ventral striatal ramp cell and a hippocampal place cell from a different subject, R184-2009-09-12, tetrode 1 cell 5 (A) and tetrode 6 cell 7 (B). Figure layout as in Figure 3.

A
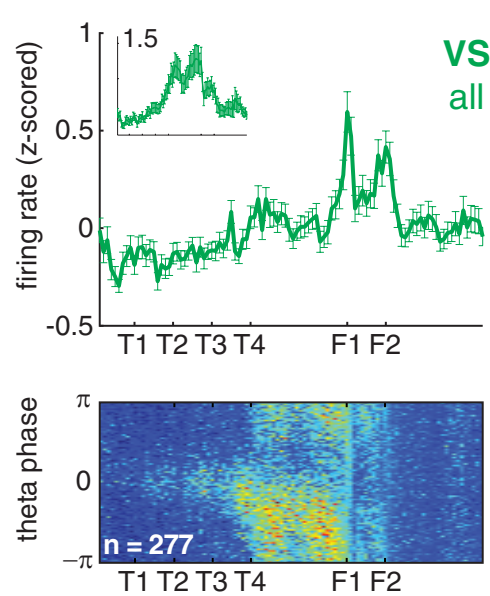

B
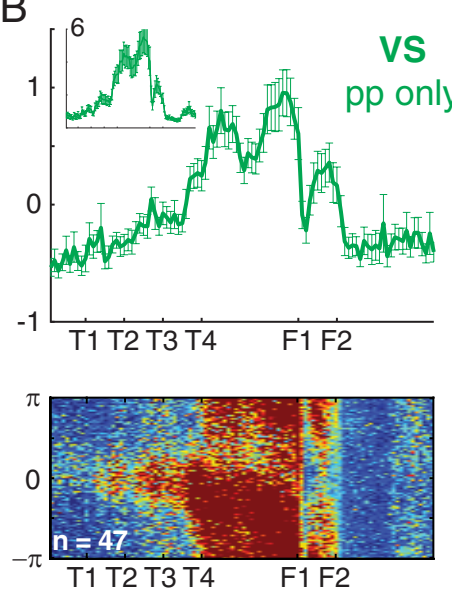

left panel). As indicated by the gradual color change, coding for theta phase, of the spikes plotted in the top left panel, as well as by the "banana plots" of phase by space, ramp cell theta phase precessed as animals approached the reward sites. Compared with hippocampal (CA1) place cell phase precession of simultaneously recorded neurons (Figs. 3, 4B), the rate of precession was much slower, as well as $\pi$ radians out of phase: note how the ventral striatal neurons start firing at zero phase and precess to between $-\pi$ and $-2 \pi$, whereas the hippocampal neurons precessed from $\pi$ to between 0 and $-\pi$ (for both areas phase was calculated relative to the same theta signal recorded from the hippocampal fissure). For comparison, we also calculated spike phase relative to the theta-band-filtered LFP recorded from the ventral striatum. Relative to this signal, there was still evidence of phase precession, albeit less clearly visible than for hippocampal theta. We address this issue in detail below.

To confirm these impressions on the population level, we plotted the average spatial tuning curves over the track; because we found no difference between left and right sides, these were averaged together. Similarly, we looked for, but did not find, systematic within-session changes in the average tuning curves, either during initial learning (Fig. $1 \mathrm{~A}$ ) or after a reward contingency switch during switch sessions (Fig. 1B). When considering all recorded neurons, the different nature of ventral striatal and hippocampal population properties is readily apparent; ventral stri-

Figure 5. Population differences in firing rate and phase precession between simultaneously recorded ventral striatal and hippocampal neurons. Top row, Average $z$-scored firing rate over the linearized track for all ventral striatal units $(\boldsymbol{A})$, ventral striatal units with a significant correlation between location on the track and theta firing phase ( $p<0.05$ uncorrected; $\boldsymbol{B})$, and all hippocampal CA1 units (C). pp, Phase precession. Z-scores were obtained for each unit individually by subtracting the mean firing rate (over locations) and dividing by the SD (over locations) and then averaging. Raw average firing rates are shown in the insets; the label indicates firing rate in hertz. Error bars are SEM over units. Bottom row, Average firing rate histograms by position on the track and theta firing phase. For each unit, these were constructed by dividing the two-dimensional spike count histogram by time spent in each bin and then averaged. Color scale is the same in all three histograms (blue to red, 0 to $0.12 \mathrm{~Hz}$ ). 
atal neurons showed a shallow ramping up to the reward sites, as well as a sharply peaked reward response (Fig. 5A, top) [consistent with van der Meer et al. (2010a), who compared CA3 and ventral striatal ensembles from separate animals]. When considering only ventral striatal units with a significant correlation between location on the track and theta phase ("phase precessing units," $p<0.05$ uncorrected, 47 of 277 recorded units or $17.0 \%$ ) (Fig. 5B, top) a more prominent ramping pattern emerged (Fig. 5b). Note how the final choice point (T4), and the two reward sites (F1 and F2) are emphasized. In contrast, population firing rates in CA1 were comparatively flat over the track (Fig. 5C, top) (in agreement with the CA3 data by van der Meer et al., 2010a). Thus, simultaneously recorded population firing rates in ventral striatum and hippocampus show a qualitatively different pattern.

Next, to assess the relationship between theta firing phase and location on the track, we constructed two-dimensional firing rate histograms over space and phase (Fig. 5, bottom row). This plot can be thought of as "expanding" the average firing rates shown above along an additional, phase dimension. The ventral striatal space-phase histograms showed a systematic relationship between theta firing phase and location on the track: phase precession is only seen past the final choice point (T4). In contrast, for simultaneously recorded hippocampal neurons, phase precession was apparent at a wider range of locations on the track, with the exception of between the reward sites in which raw firing rates were much lower (an effect also seen for CA3 data in the study by van der Meer et al., 2010a). In addition, differences in the speed and range of ventral striatal phase precession apparent in the examples were also present here. As with the ramp in the population firing rates above, this pattern became even more pronounced when only phase precessing units were considered (Fig. 5B, middle). Thus, at the population level, theta phase precession in ventral striatum is biased toward the reward locations, whereas simultaneously recorded neurons from hippocampus precessed at a wider range of locations.

True phase precession relative to the theta rhythm implies that spiking activity shows some degree of theta modulation, which should be slightly faster than the frequency of the reference LFP (we investigate this relative frequency issue in detail below). In line with previous reports (Tabuchi et al., 2000; Berke et al., 2004; Lansink et al., 2009) and as illustrated by the example units in Figures 3 and 4, we found clear examples of theta-modulated firing in ventral striatum. Following Royer et al. (2010), we quantified the degree of theta modulation by a multivariate fit to
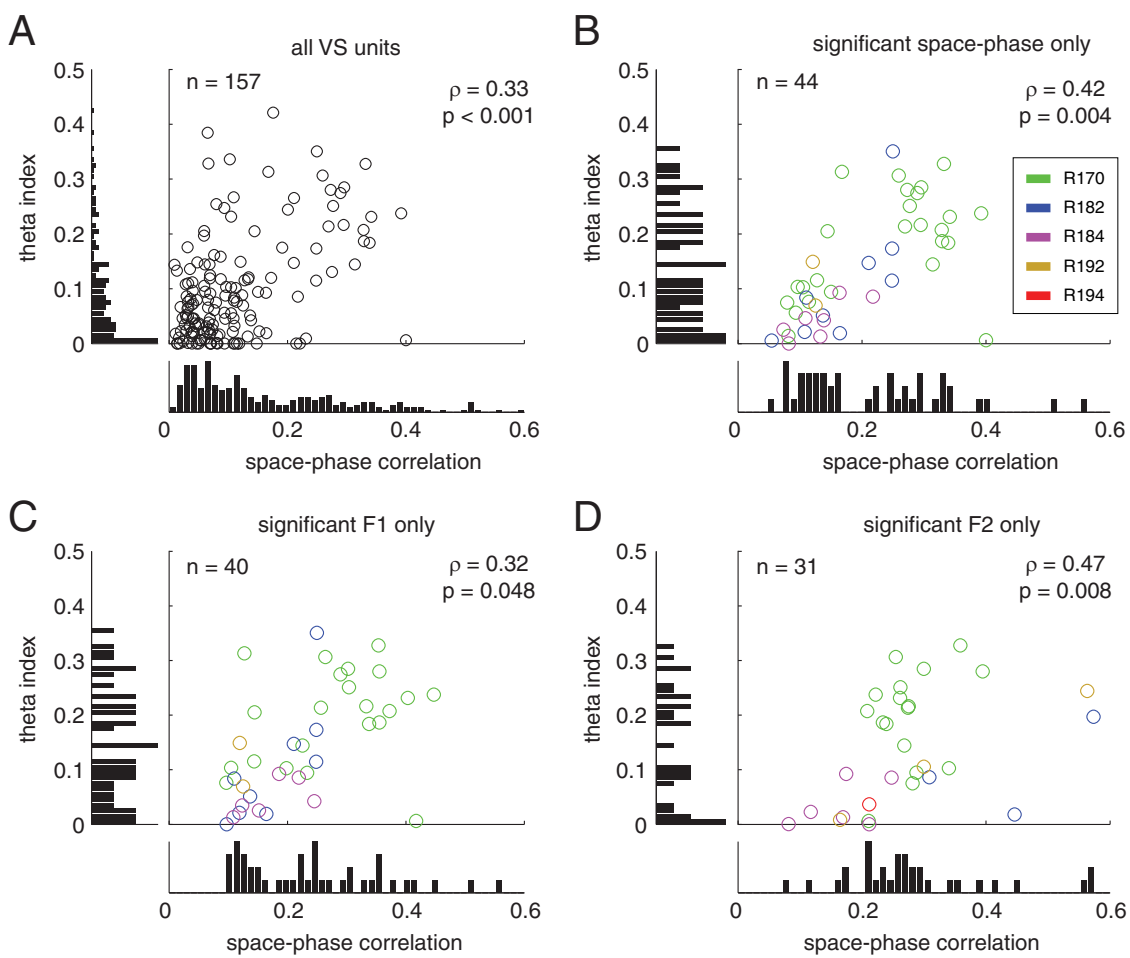

Figure 6. Theta modulation in ventral striatal units is positively correlated with space-phase correlation, a measure of phase precession strength. Plots show the marginal and joint distributions of the theta index (a measure of theta modulation obtained from multivariate fits to the autocorrelogram) and space-phase correlation. These two quantities were positively correlated regardless of whether all units $(\boldsymbol{A})$, units that phase precessed anywhere on the track $(\boldsymbol{B})$, or units that only precessed up to the first $(\boldsymbol{C})$ or second ( $\boldsymbol{D} ; \mathrm{F} 1$ and $\mathrm{F} 2$, respectively) reward site were considered.
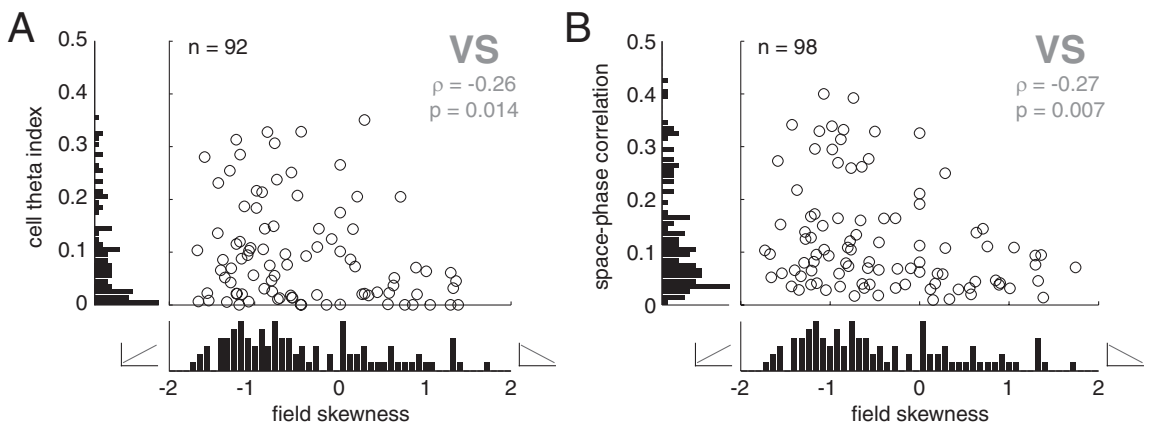

Figure 7. Ventral striatal units with a firing rate ramp up to the first reward site were preferentially theta modulated and phase precessing. To quantify the degree of "rampiness," the skewness of firing rate over space was used; negative numbers indicate an upward-sloping ramp as animals approach the reward site, and positive skewness indicates a downward-sloping ramp. To identify ramp cell candidates, only data points for which the spatial tuning curve was well fit by linear regression $(p<0.05)$ are shown; note that this criterion does not contain a bias in the upward- or downward-sloping direction. Nevertheless, the population of ventral striatal units was biased in the negative skewness/upward-ramping direction $(\boldsymbol{A}, \boldsymbol{B})$. Upward ramping was associated with higher theta modulation and stronger phase precession.

the autocorrelogram. Over the population of ventral striatal units, higher theta modulation (as quantified by the theta index obtained by fitting the autocorrelogram) was associated with higher space-phase correlations (indicating phase precession strength, $\rho=0.33, p<0.001$ ) (Fig. $6 A$ ). This was the case regardless of whether all units or only significantly phase precessing units were considered (Fig. 6B-D). The distributions of theta modulation index and space-phase correlation did not appear to be multimodal, suggesting that these quantities may vary continuously rather than be a discrete "on-off" property of ventral striatal neurons. 



Figure 8. Relationship between firing rate and theta phase in ventral striatal ramp neurons. $A$, Phase/rate scatter plots for the example neuron in Figure 3. Each data point in the plots corresponds to one bin in the tuning curve of the neuron over the (linearized) track. Left, Phase/rate scatter plot for the firing rate ramp up to the first reward site (F1); the color of each dot corresponds to position along the ramp. Note the clear phase precession (from 0 to approximately $-\pi$ radians) as the reward site is approached (color change from blue to green). Middle, Phase/rate scatter plot for the firing rate ramp up to the second reward site (F2). Note the much shorter, but clearly visible, sequence of phase precessing data points in the bottom left of the plot. Right, The F1 and F2 firing rate ramps occupy a distinct area of the phase/rate plot: the same theta phase (e.g., $-\pi / 2$ ) is associated with different firing rates for two ramps. This can also be seen in the example phase plot in Figure 3 , top right. $\boldsymbol{B}$, This pattern held across all phase precessing neurons $(n=47)$. For clarity, only spatial bins for which theta firing phase could be estimated with $95 \%$ confidence intervals equal to or smaller than $\pi / 4$ were included in both plots, and the range of spatial locations included was matched for the F1 and F2 ramps; see the schematics above each scatter plot for included regions.

Given the tendency of the ventral striatal population average firing rate to ramp up to the reward sites, we investigated the relationship between the shape of spatial tuning curves of the individual units and their theta modulation. First, to confirm the presence of a population of ramp cells, we applied a linear regression analysis to the spatial tuning curve of each unit. The distribution of units with a significant regression fit was biased in the "ramp up" direction (Fig. 7A, negative skewness numbers correspond to "up" ramps). For clarity, the data shown in Figure 7 were restricted to regression fits from the start point on the track to the first reward site (Figs. 3, 4, "S" to "F1"). Thus, the overall ramp shape in the population average is accompanied by a bias toward up ramps in individual neurons. As the scatter plot in Figure $7 A$ shows, such ramping units tended to be associated with stronger theta modulation $(\rho=-0.26, p=0.014)$. Furthermore, up ramping units had stronger phase precession $(\rho=$ $-0.27, p=0.007$ ) (Fig. $7 B$ ). Thus, anticipatory ramping activity in ventral striatum is not simply one of a distribution of patterns; rather, it is a privileged firing pattern, specifically associated with higher theta modulation and phase precession on this task.

It is known that hippocampal place fields show a particular relationship between theta firing phase and firing rate, which can inform mechanistic explanations for the phase precession effect (Harris et al., 2002; Mehta et al., 2002). As shown above, phase precessing neurons in ventral striatum tended to show excitatory ramping, with firing rates increasing as rats approached the reward sites. This observation raises the possibility that, as suggested by the model proposed by Mehta et al. (2002), ventral striatal firing phase precession results from a ramp in instanta- neous firing rate. To test this idea, we constructed scatter plots of firing phase against firing rate, with each point corresponding to average firing rate and average theta firing phase in one spatial bin (Mehta et al., 2002). For the example neuron in Figure 3, the banana plots appear to show a clear correlation between firing rate and theta phase as firing rate increases up to the first reward site (F1). Similarly, firing rate and phase appear correlated when approaching the second reward site (F2); note, however, that although the range of theta phase precession for the two reward sites appears similar (Fig. 3, top right), the firing rates for the F1 and F2 ramps are quite different. In the phase/rate scatter plot for this neuron (Fig. 8A), this difference can be seen by comparing the magenta points (corresponding to the $\mathrm{F} 1 \mathrm{ramp}$ ) and the cyan points (F2 ramp). In effect, the ramps for the first and second reward sites cover distinct areas of the phase/rate space. When data from all ventral striatal phase precessing neurons was combined (Fig. $8 \mathrm{~B}$ ), this separation persisted; note, for instance, how there are very few cyan (F2) points in the top left area of the plot. Thus, although within firing rate ramps there was a clear relationship between ventral striatal firing rate and theta firing phase, this relationship was not constant between ramps.

\section{Relationship between hippocampal and ventral striatal theta}

An important question arising from the observation of theta phase precession in ventral striatum is whether this phenomenon is better accounted for by reference to the local (striatal) theta rhythm or by reference to the hippocampal theta rhythm. We thus sought to compare the properties of the theta rhythm and theta phase precession for these different reference signals. In 
general, theta band oscillations were more prominent in the hippocampus fissure than in ventral striatum, both as measured by their overall power and by the presence of a distinct peak in the power spectrum density separating the theta band from adjacent frequencies (Fig. 9A). Estimating average theta frequency yielded no significant difference between the two sites, although there was a tendency for the ventral striatal theta to be slower (Fig. 9B). As noted previously, true phase precession implies that the theta modulation in the spiking of the unit is faster than that of the global (reference) theta signal. We thus plotted these quantities in a scatter plot for both the ventral striatal (Fig. 10A) and hippocampal reference (Fig. $10 \mathrm{~B}$ ). In both cases, ventral striatal units fell above the diagonal, corresponding to a faster spiking modulation than global modulation. We observed a nearly bimodal distribution of global hippocampal theta frequencies (Fig. $10 \mathrm{~B}$, bottom bar plot), raising the possibility that a large number of apparently phase precessing units were artifactually precessing because of an unusually slow theta signal used as a reference. However, we rule out this possibility based on the observation that phase precession remains relative to the ventral striatal theta rhythm (see example in Fig. 3, which was taken from a "slow" hippocampal theta session) and that even for the "fast" hippocampal theta sessions, spiking theta modulation is faster still (Fig. $10 \mathrm{~B}$ ). As shown in Figure 10, $C$ and $D$, however, phase precession tended to be clearer (stronger space-phase correlation) to the hippocampal theta rhythm compared with ventral striatal theta.

The power of recorded field potentials depends on several factors, including the geometry and alignment of the neurons contributing to it (Johnston and $\mathrm{Wu}$, 1995). Thus, a theoretical possibility is that underlying the relatively weak ventral striatal theta LFP is a strongly thetamodulated population, albeit one that is not arranged in a neat layered form conducive to generating a large theta field as occurs in the hippocampus. To test for this possibility, we compared the average autocorrelograms of neurons in ventral striatum and hippocampus (Fig. 11). In contrast to the strongly theta-modulated putative pyramidal neurons and inhibitory interneurons in the hippocampus (bottom row), theta modulation in ventral striatal neuron spiking was barely visible in the average autocorrelograms (top row). Thus, in line with the relatively weak theta component of the ventral striatal LFP, the population of ventral striatal neurons displayed little theta modulation in their spiking activity.

The hippocampal formation sends a projection to the ventral striatum, providing a possible anatomical basis for ventral striatal units to receive theta-modulated and/or phase-precessing input. Given the tendency for ventral striatal phase precession to occur at specific parts of the track (on reward approach) (Fig. $5 B$ ), we asked whether theta activity in both tended to co-occur at particular places; this would indicate a possible functional interaction based on theta synchronization. We plotted the theta band coherence over the spatial extent of the track (Fig. 12A,B) and


Figure 9. Comparison of ventral striatal and hippocampal local field potential properties. $\boldsymbol{A}$, Average PSD for HC (recorded from the fissure) and VS. Note the prominent theta peak and harmonics in the hippocampal PSD compared with a barely noticeable "shoulder" in the ventral striatal data. Ventral striatum contained clear low $(50 \mathrm{~Hz})$ and high $(70-100 \mathrm{~Hz})$ gamma power (in line with van der Meer et al., 2010b) absent from the hippocampal fissure. $\boldsymbol{B}$, Average theta frequency was not different between the two structures.
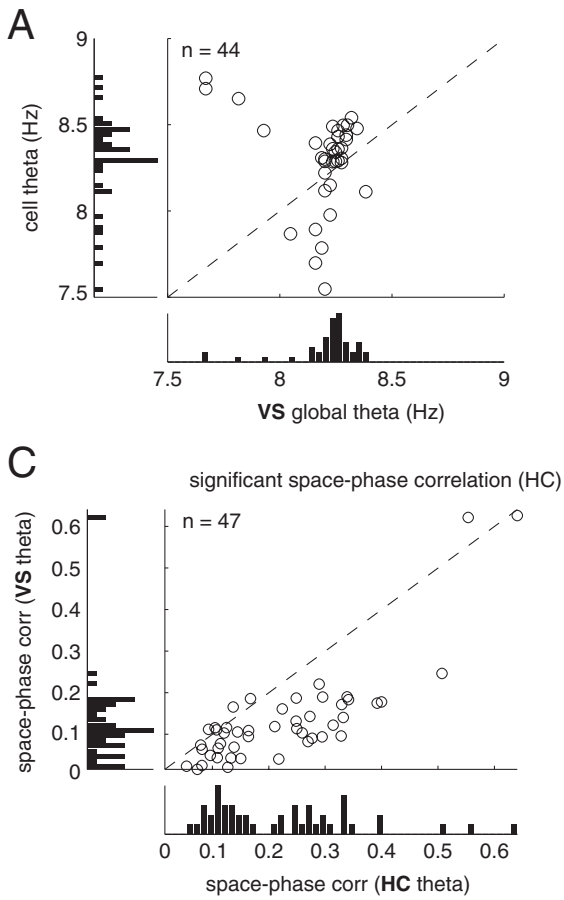
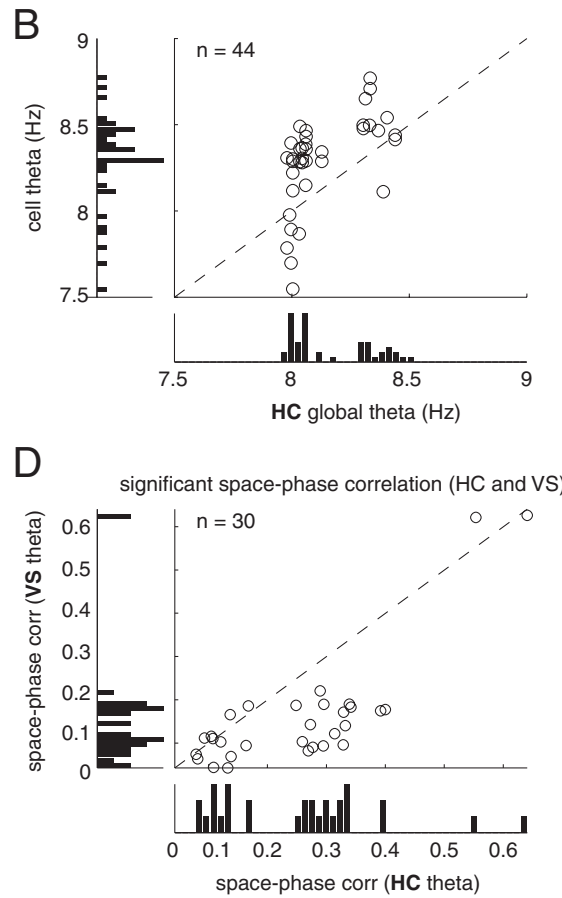

Figure 10. Comparison of phase precession relative to ventral striatal and hippocampal theta signals. Units with a significant space-phase correlation ("phase precessing units") had a faster theta modulation in their spiking compared with the frequency of both the ventral striatal $(\boldsymbol{A})$ and hippocampal $(\boldsymbol{B})$ reference signal. Phase precession strength tended to be stronger relative to the hippocampal, compared with ventral striatal, reference signal $(\boldsymbol{C})$, this was true even when only cells that phase precessed to both the hippocampal and ventral striatal theta rhythm were considered $(\boldsymbol{D})$.

found that, although the pattern looked somewhat different depending on whether coherence was calculated relative to the fissure or layer electrodes in hippocampus, in both cases coherence tended to ramp up to the reward sites. This temporal cooccurrence of theta power supports the notion that phase precession in ventral striatum arises from an interaction between hippocampus and ventral striatum rather than from independent theta sources in the two structures.

\section{Discussion}

Anticipatory ramp neurons in ventral striatum phase precess relative to the hippocampal theta rhythm. The phase of these neurons was preferentially coupled to hippocampal theta phase 


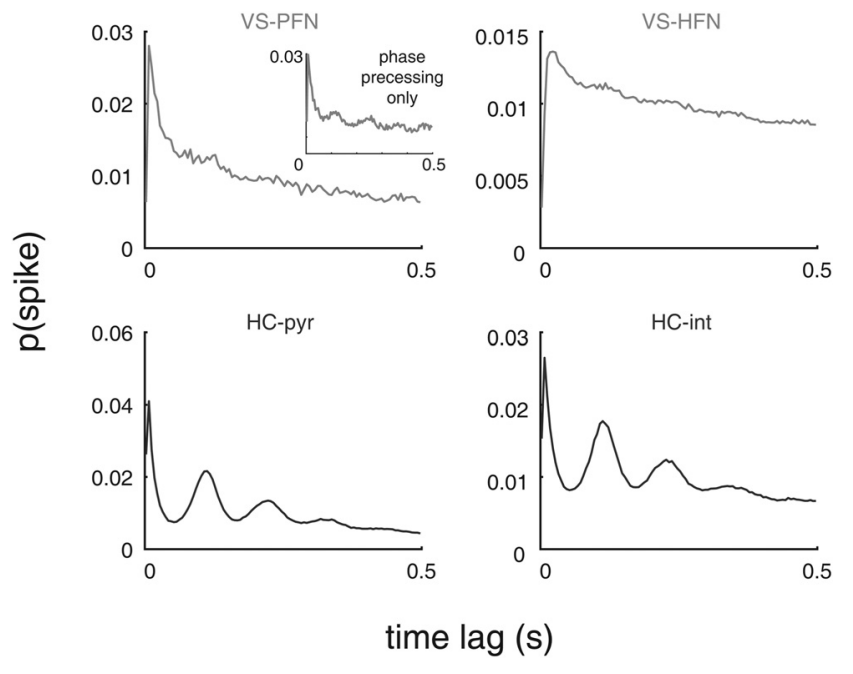

Figure 11. Relatively weak theta modulation in ventral striatal spiking. Shown are the average autocorrelograms for phasically firing neurons (PFN) (putative medium spiny neurons) and high-firing neurons (HFN) (putative fast-spiking interneurons) in VS (top row) and for putative hippocampal pyramidal neurons (pyr) and interneurons (int; $\mathrm{HC}$, bottom row). Note how the clear theta modulation in the hippocampal neurons is nearly absent from the ventral striatal population. Even if only phase precessing ventral striatal neurons are considered (top left, inset), theta modulation is noticeably weaker than that in hippocampal neurons. Autocorrelograms were constructed by counting the number of spike pairs at a given time lag (in $5 \mathrm{~ms}$ bins) and normalizing by the total number of counts in the $500 \mathrm{~ms}$ window shown.

A
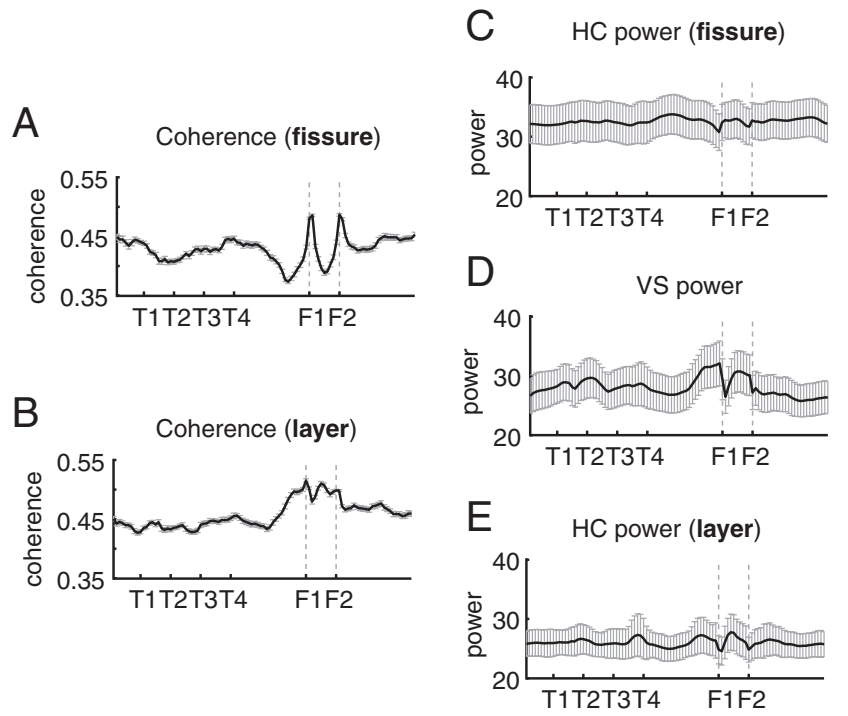

Figure 12. Theta band coherence between hippocampus and ventral striatum ramped up to the reward sites, both when coherence was computed relative to the hippocampal fissure electrode $(\boldsymbol{A})$ or an electrode in or near the CA1 cell layer $(\boldsymbol{B})$. For comparison, the spatial distributions of theta band power are also shown for each recording location separately $(\boldsymbol{C}-\boldsymbol{E})$.

rather than a local ventral striatal theta phase signal. Phase precession was not a general property of ventral striatal neurons but was specific to a subset of ventral striatal neurons with a ramping tuning curve that consistently increased with spatial approach to reward. In contrast, simultaneously recorded CA1 cells showed uniformly distributed spatial tuning curves, which precessed locally through their given place fields. Phase precession to local theta rhythms has been seen in the hippocampal formation (O'Keefe and Recce 1993; Skaggs et al., 1996 and many others) (for review, see Maurer and McNaughton, 2007) and in entorhinal cortex (Hafting et al., 2008). Phase precession to hippocampal theta has also been found in prefrontal cortex (Jones and Wilson, 2005a) but has to our knowledge not been established elsewhere. These data imply that precise spike timing is also an important property of striatal processing and suggest that ventral striatum participates in the same temporal tapestry as hippocampus.

The precise coordination in spike timing apparent from striatal phase precession relative to the hippocampal theta rhythm suggests that the two phenomena are not independent. The known anatomical projection from the hippocampal formation to the ventral striatum likely provides a basis for this coordination (Groenewegen et al., 1987; Finch, 1996; Voorn et al., 2004; Humphries and Prescott, 2010). Thus, one possible conception of how striatal phase precession arises is that it is "inherited" from hippocampal inputs that themselves already phase precess. At one extreme of this idea would be the possibility that the activity of a phase precessing neuron in ventral striatum is a copy (subject to delays and transmission noise) of the activity of a single hippocampal neuron. Somewhat less restrictively, phase precessing ventral striatal neurons may receive a number of hippocampal or other inputs, some of which already phase precess. At the other end of the spectrum, a ventral striatal neuron may phase precess as a result of local dynamics such as subthreshold membrane oscillations or inhibitory feedback. We discuss these possibilities in turn.

Could phase precessing ventral striatal neurons be simply reproducing hippocampal output? This hypothesis would require (1) a hippocampal neuron with the same ramping tuning curve observed for ventral striatal phase precessing neurons and (2) a relationship between firing rate and phase inconsistent with that known to occur in hippocampal place cells. We simultaneously recorded dorsal CA1 ensembles and found clear differences in spatial tuning and phase precession between the two structures. It is possible that the inputs to the phase precessing ventral striatal neurons may arise from the ventral or intermediate instead of the dorsal hippocampus (Groenewegen et al., 1987; Humphries and Prescott, 2010). The fact that we observed striatal phase precession $180^{\circ}$ out-of-phase with simultaneously recorded dorsal hippocampal phase precession is consistent with this idea (the ventral hippocampal theta rhythm is $\sim 180^{\circ}$ out-of-phase compared with dorsal) (Lubenov and Siapas, 2009; Royer et al., 2010). However, Royer et al. did not find clear phase precession in ventral hippocampal cells, despite recording on similar track-based mazes. In addition, hippocampal neurons tend to have a characteristic relationship between firing rate and phase (Harris et al., 2002; Mehta et al., 2002). For ventral striatal ramp neurons, we found that, although such a relationship was present in firing rate ramps up to different reward sites separately, it was not constant across different reward sites. This observation can, however, be reconciled with the excitatory ramp-inhibitory oscillation mechanism suggested by Mehta et al. (2002) if we assume that a ventral striatal ramp neuron receives input from two distinct populations of hippocampal neurons rather than "copy" a single hippocampal source.

A different possibility is that theta phase effects in ventral striatum are independent of the hippocampal theta rhythm but are locally generated instead. A number of mechanisms for the generation of theta phase precession in the hippocampus have been proposed; virtually all of these require some locally generated theta source. For instance, "dual-oscillator" type proposals rely on an interaction between two theta rhythms of slightly differing frequencies, either generated in the dendrites and the soma or by membrane and network mechanisms, respectively (Harris et al., 2002; O'Keefe and Burgess, 2005; Maurer and Mc- 
Naughton, 2007; Harvey et al., 2009; Losonczy et al., 2010). A slightly different proposal by Mehta et al. (2002) requires a thetaoscillating inhibition interacting with ramping excitation. In our recordings, theta modulation in ventral striatum was weak compared with that in the hippocampus, at the LFP, putative projection neuron, and interneuron levels. Furthermore, in vitro studies in ventral striatum have not found systematic properties, such as subthreshold oscillations, that could support the local generation of theta activity in the striatum (Goto and O'Donnell, 2001; Bracci et al., 2003; Taverna et al., 2007). Thus, we suggest that local generation of theta phase precession in ventral striatum is unlikely.

Instead, phase precession in ventral striatum may derive from learned associations between populations of hippocampal inputs and reward-related activity. This hypothesis depends on a translation from potentially uniformly distributed place fields in such a way to systematically bias phase precession toward the observed ramp pattern leading to reward sites on the track; note that this pattern is different from the experience-dependent asymmetric ramping known to develop with experience in hippocampal place fields (Mehta et al., 1997). Our results suggest the possibility that phase precessing ventral striatal ramp neurons preferentially receive input from those hippocampal neurons that were active leading up to reward sites. This connectivity could arise via the actions of the neurotransmitter dopamine, which ventral striatum receives from the ventral tegmental area and which may contribute to a trisynaptic learning rule strengthening those hippocampal inputs specifically active around the time of (unexpected) reward. Selection of hippocampal inputs along these lines could accomplish the association of place and reward thought to underlie place preference and directed movement initiation (Pennartz et al., 1994; Ito et al., 2008). Of course, such a mechanism would not preclude interactions with other inputs converging on the ventral striatum, such as those from prefrontal cortical areas (where theta rhythms and phase locking are also prominent) (Jones and Wilson 2005a,b; Siapas et al., 2005; Hyman et al., 2010; van Wingerden et al., 2010).

Within the hippocampal formation, phase precession and the temporal sequence compression it implies when places or events are sequentially experienced is thought to underlie rapid encoding of experience (Skaggs et al., 1996; Lisman, 2005; Dragoi and Buzsáki, 2006; Morris, 2006; Maurer and McNaughton, 2007). The observation that ventral striatal neurons phase precess extends this idea to associations and sequences beyond the hippocampus. In support of this possibility, Lansink et al. (2009) found that offline reactivation of hippocampal-ventral striatum cell pairs was strongest when both cells showed theta modulation. Our finding of theta phase precession in ventral striatum provides a potential explanation for this effect through the idea that hippocampally driven associative learning contains at least two distinct stages: sequence compression arising from phase precession allows for rapid initial encoding, and subsequent offline "replay" may serve to selectively strengthen those initial traces in a consolidation process (Nadel et al., 2000; Tse et al., 2007; Girardeau et al., 2009; Lansink et al., 2009; Ego-Stengel and Wilson, 2010).

What information is coded by phase precessing neurons in ventral striatum? Phase precessing neurons were most likely to ramp up in firing rate to the reward sites, a pattern clearly apparent in the population average. Previous studies investigating the properties of such ramp cells have similarly found relationships to reward delivery across different tasks, species, and reward types (Schultz et al., 1992; Carelli and Deadwyler, 1994; Lavoie and Mizumori, 1994; Miyazaki et al., 1998; Cromwell and Schultz, 2003; Khamassi et al., 2008; van der Meer and Redish, 2009a). The disconnection study by Ito et al. (2008) identified a specific impairment of $\mathrm{HC}$-nucleus accumbens shell disconnections in a conditioned place preference task, although the same subjects were not impaired in simple pavlovian cue discrimination. These results are congruent with the notion of Mogenson et al. (1980) that the ventral striatum provides a limbic-motor interface. In this case, the HC-VS association could imbue particular places with motivational relevance. Ito et al. (2008) did not distinguish between the learning and performance stages of the place preference task, raising the question of whether the HC-VS association is only important for learning or whether it also plays a direct role in decision-making performance (Atallah et al., 2007).

A number of studies have shown ventral striatal activity at or preceding the time of decision, specific to particular actions or goals (German and Fields, 2007; Roesch et al., 2009; van der Meer and Redish, 2009a), and, in general, ventral striatum is a critical structure for the expression of a large number of motivated behaviors (Pennartz et al., 1994; Cardinal et al., 2002; Schoenbaum and Setlow, 2003; Nicola, 2007; Humphries and Prescott, 2010). In the hippocampal field, it has been noted that theta phase coding not only has implications for learning but could also be exploited to obtain a more accurate spatial readout (O'Keefe and Recce, 1993; Skaggs et al., 1996; Tsodyks et al., 1996; Jensen and Lisman, 2000; Huxter et al., 2003; Nadasdy, 2010). Similarly, given the strong bias of phase precessing ventral striatal neurons to the reward sites, spiking phase of the ventral striatal neurons could provide a readout of distance or time to reward. More generally, phase precession in ventral striatum thus not only suggests a mechanism for associating place and reward information but may also have consequences for how information is coded in ventral striatum.

\section{References}

Adhikari A, Topiwala MA, Gordon JA (2010) Synchronized activity between the ventral hippocampus and the medial prefrontal cortex during anxiety. Neuron 65:257-269.

Atallah HE, Lopez-Paniagua D, Rudy JW, O’Reilly RC (2007) Separate neural substrates for skill learning and performance in the ventral and dorsal striatum. Nat Neurosci 10:126-131.

Bast T, Feldon J (2003) Hippocampal modulation of sensorimotor processes. Prog Neurobiol 70:319-345.

Benchenane K, Peyrache A, Khamassi M, Tierney PL, Gioanni Y, Battaglia FP, Wiener SI (2010) Coherent theta oscillations and reorganization of spike timing in the hippocampal-prefrontal network upon learning. Neuron 66:921-936.

Berke JD, Okatan M, Skurski J, Eichenbaum HB (2004) Oscillatory entrainment of striatal neurons in freely moving rats. Neuron 43:883-896.

Bi GQ, Poo MM (1998) Synaptic modifications in cultured hippocampal neurons: dependence on spike timing, synaptic strength, and postsynaptic cell type. J Neurosci 18:10464-10472.

Bracci E, Centonze D, Bernardi G, Calabresi P (2003) Voltage-dependent membrane potential oscillations of rat striatal fast-spiking interneurons. J Physiol 549:121-130.

Cardinal RN, Parkinson JA, Hall J, Everitt BJ (2002) Emotion and motivation: the role of the amygdala, ventral striatum, and prefrontal cortex. Neurosci Biobehav Rev 26:321-352.

Carelli RM, Deadwyler SA (1994) A comparison of nucleus accumbens neuronal firing patterns during cocaine self-administration and water reinforcement in rats. J Neurosci 14:7735-7746.

Cromwell HC, Schultz W (2003) Effects of expectations for different reward magnitudes on neuronal activity in primate striatum. J Neurophysiol 89:2823-2838.

Csicsvari J, Hirase H, Czurkó A, Mamiya A, Buzsáki G (1999) Oscillatory 
coupling of hippocampal pyramidal cells and interneurons in the behaving rat. J Neurosci 19:274-287.

Daw N (2003) Reinforcement learning models of the dopamine system and their behavioral implications. PhD thesis, Carnegie Mellon University.

Dragoi G, Buzsáki G (2006) Temporal encoding of place sequences by hippocampal cell assemblies. Neuron 50:145-157.

Ego-Stengel V, Wilson MA (2010) Disruption of ripple-associated hippocampal activity during rest impairs spatial learning in the rat. Hippocampus 20:1-10.

Eichenbaum H, Dudchenko P, Wood E, Shapiro M, Tanila H (1999) The hippocampus, memory, and place cells: is it spatial memory or a memory space? Neuron 23:209-226.

Finch DM (1996) Neurophysiology of converging synaptic inputs from the rat prefrontal cortex, amygdala, midline thalamus, and hippocampal formation onto single neurons of the caudate/putamen and nucleus accumbens. Hippocampus 6:495-512.

German PW, Fields HL (2007) Rat nucleus accumbens neurons persistently encode locations associated with morphine reward. J Neurophysiol 97:2094-2106.

Girardeau G, Benchenane K, Wiener SI, Buzsáki G, Zugaro MB (2009) Selective suppression of hippocampal ripples impairs spatial memory. Nat Neurosci 12:1222-1223.

Goto Y, O'Donnell P (2001) Synchronous activity in the hippocampus and nucleus accumbens in vivo. J Neurosci 21:RC131(1-5).

Groenewegen HJ, Vermeulen-Van der Zee E, te Kortschot A, Witter MP (1987) Organization of the projections from the subiculum to the ventral striatum in the rat. a study using anterograde transport of phaseolus vulgaris leucoagglutinin. Neuroscience 23:103-120.

Gupta AS, van der Meer MA, Touretzky DS, Redish AD (2010) Hippocampal replay is not a simple function of experience. Neuron 65:695-705.

Hafting T, Fyhn M, Bonnevie T, Moser MB, Moser EI (2008) Hippocampus-independent phase precession in entorhinal grid cells. Nature 453:1248-1252.

Harris KD, Henze DA, Hirase H, Leinekugel X, Dragoi G, Czurkó A, Buzsáki G (2002) Spike train dynamics predicts theta-related phase precession in hippocampal pyramidal cells. Nature 417:738-741.

Harvey CD, Collman F, Dombeck DA, Tank DW (2009) Intracellular dynamics of hippocampal place cells during virtual navigation. Nature 461:941-946.

Humphries MD, Prescott TJ (2010) The ventral basal ganglia, a selection mechanism at the crossroads of space, strategy, and reward. Prog Neurobiol 90:385-417.

Huxter J, Burgess N, O'Keefe J (2003) Independent rate and temporal coding in hippocampal pyramidal cells. Nature 425:828-832.

Hyman JM, Zilli EA, Paley AM, Hasselmo ME (2010) Working memory performance correlates with prefrontal-hippocampal theta interactions but not with prefrontal neuron firing rates. Front Integr Neurosci 4:2.

Ito R, Robbins TW, Pennartz CM, Everitt BJ (2008) Functional interaction between the hippocampus and nucleus accumbens shell is necessary for the acquisition of appetitive spatial context conditioning. J Neurosci 28:6950-6959.

Jensen O, Lisman JE (2000) Position reconstruction from an ensemble of hippocampal place cells: contribution of theta phase coding. J Neurophysiol 83:2602-2609.

Johnson A, Redish AD (2007) Neural ensembles in CA3 transiently encode paths forward of the animal at a decision point. J Neurosci 27:12176-12189.

Johnston D, Wu SM (1995) Foundations of cellular neurophysiology. Cambridge, MA: Bradford Books.

Jones MW, Wilson MA (2005a) Phase precession of medial prefrontal cortical activity relative to the hippocampal theta rhythm. Hippocampus 15:867-873.

Jones MW, Wilson MA (2005b) Theta rhythms coordinate hippocampalprefrontal interactions in a spatial memory task. PLoS Biol 3:e402.

Khamassi M, Mulder AB, Tabuchi E, Douchamps V, Wiener SI (2008) Anticipatory reward signals in ventral striatal neurons of behaving rats. Eur J Neurosci 28:1849-1866.

Lansink CS, Goltstein PM, Lankelma JV, McNaughton BL, Pennartz CM (2009) Hippocampus leads ventral striatum in replay of place-reward information. PLoS Biol 7:e1000173.

Lavoie AM, Mizumori SJ (1994) Spatial, movement-and reward-sensitive discharge by medial ventral striatum neurons of rats. Brain Res 638:157-168.

Lisman J (2005) The theta/gamma discrete phase code occuring during the hippocampal phase precession may be a more general brain coding scheme. Hippocampus 15:913-922.

Losonczy A, Zemelman BV, Vaziri A, Magee JC (2010) Network mechanisms of theta related neuronal activity in hippocampal CAl pyramidal neurons. Nat Neurosci 13:967-972.

Lubenov EV, Siapas AG (2009) Hippocampal theta oscillations are travelling waves. Nature 459:534-539.

Martin PD (2001) Locomotion towards a goal alters the synchronous firing of neurons recorded simultaneously in the subiculum and nucleus accumbens of rats. Behav Brain Res 124:19-28.

Maurer AP, McNaughton BL (2007) Network and intrinsic cellular mechanisms underlying theta phase precession of hippocampal neurons. Trends Neurosci 30:325-333.

Maurer AP, Cowen SL, Burke SN, Barnes CA, McNaughton BL (2006) Phase precession in hippocampal interneurons showing strong functional coupling to individual pyramidal cells. J Neurosci 26:13485-13492.

Mehta MR, Barnes CA, McNaughton BL (1997) Experience-dependent, asymmetric expansion of hippocampal place fields. Proc Natl Acad Sci U S A 94:8918-8921.

Mehta MR, Quirk MC, Wilson MA (2000) Experience-dependent asymmetric shape of hippocampal receptive fields. Neuron 25:707-715.

Mehta MR, Lee AK, Wilson MA (2002) Role of experience and oscillations in transforming a rate code into a temporal code. Nature 417:741-746.

Mitra P, Bokil H (2008) Observed brain dynamics. New York: Oxford UP.

Miyazaki K, Mogi E, Araki N, Matsumoto G (1998) Reward-quality dependent anticipation in rat nucleus accumbens. Neuroreport 9:3943-3948.

Mogenson GJ, Jones DL, Yim CY (1980) From motivation to action: functional interface between the limbic system and the motor system. Prog Neurobiol 14:69-97.

Morris RG (2006) Elements of a neurobiological theory of hippocampal function: the role of synaptic plasticity, synaptic tagging and schemas. Eur J Neurosci 23:2829-2846.

Nadasdy Z (2010) Binding by asynchrony: the neuronal phase code. Front Neurosci 4:51.

Nadel L, Samsonovich A, Ryan L, Moscovitch M (2000) Multiple trace theory of human memory: computational, neuroimaging, and neuropsychological results. Hippocampus 10:352-368.

Nicola SM (2007) The nucleus accumbens as part of a basal ganglia action selection circuit. Psychopharmacology (Berl) 191:521-550.

O'Keefe J, Burgess N (2005) Dual phase and rate coding in hippocampal place cells: theoretical significance and relationship to entorhinal grid cells. Hippocampus 15:853-866.

O'Keefe J, Nadel L (1978) The hippocampus as a cognitive map. Oxford: Clarendon.

O'Keefe J, Recce ML (1993) Phase relationship between hippocampal place units and the EEG theta rhythm. Hippocampus 3:317-330.

Pastalkova E, Itskov V, Amarasingham A, Buzsáki G (2008) Internally generated cell assembly sequences in the rat hippocampus. Science 321:1322-1327.

Paxinos G, Watson C (1998) The rat brain in stereotaxic coordinates, Ed 4. San Diego: Academic.

Pennartz CM, Groenewegen HJ, Lopes da Silva FH (1994) The nucleus accumbens as a complex of functionally distinct neuronal ensembles: an integration of behavioural, electrophysiological and anatomical data. Prog Neurobiol 42:719-761.

Percival DB, Walden AT (1993) Spectral analysis for physical applications. Cambridge, UK: Cambridge UP.

Peyrache A, Khamassi M, Benchenane K, Wiener SI, Battaglia FP (2009) Replay of rule-learning related neural patterns in the prefrontal cortex during sleep. Nat Neurosci 12:919-926.

Ranck JB Jr (1973) Studies on single neurons in dorsal hippocampal formation and septum in unrestrained rats. I. Behavioral correlates and firing repertoires. Exp Neurol 41:461-531.

Redish AD (1999) Beyond the cognitive map. Cambridge, MA: Massachusetts Institute of Technology.

Robbe D, Buzsáki G (2009) Alteration of theta timescale dynamics of hippocampal place cells by a cannabinoid is associated with memory impairment. J Neurosci 29:12597-12605.

Roesch MR, Singh T, Brown PL, Mullins SE, Schoenbaum G (2009) Ventral 
striatal neurons encode the value of the chosen action in rats deciding between differently delayed or sized rewards. J Neurosci 29:13365-13376.

Royer S, Sirota A, Patel J, Buzsáki G (2010) Distinct representations and theta dynamics in dorsal and ventral hippocampus. J Neurosci 30:1777-1787.

Schmidt R, Diba K, Leibold C, Schmitz D, Buzsáki G, Kempter R (2009) Singletrial phase precession in the hippocampus. J Neurosci 29:13232-13241.

Schmitzer-Torbert N, Redish AD (2004) Neuronal activity in the rodent dorsal striatum in sequential navigation: separation of spatial and reward responses on the multiple t task. J Neurophysiol 91:2259-2272.

Schmitzer-Torbert NC, Redish AD (2008) Task-dependent encoding of space and events by striatal neurons is dependent on neural subtype. Neuroscience 153:349-360.

Schoenbaum G, Setlow B (2003) Lesions of nucleus accumbens disrupt learning about aversive outcomes. J Neurosci 23:9833-9841.

Schultz W, Apicella P, Scarnati E, Ljungberg T (1992) Neuronal activity in monkey ventral striatum related to the expectation of reward. J Neurosci 12:4595-4610.

Siapas AG, Lubenov EV, Wilson MA (2005) Prefrontal phase locking to hippocampal theta oscillations. Neuron 46:141-151.

Skaggs WE, McNaughton BL, Wilson MA, Barnes CA (1996) Theta phase precession in hippocampal neuronal populations and the compression of temporal sequences. Hippocampus 6:149-172.

Swanson LW (1981) A direct projection from Ammon's horn to prefrontal cortex in the rat. Brain Res 217:150-154.

Tabuchi ET, Mulder AB, Wiener SI (2000) Position and behavioral modulation of synchronization of hippocampal and accumbens neuronal discharges in freely moving rats. Hippocampus 10:717-728.

Taverna S, Canciani B, Pennartz CM (2007) Membrane properties and synaptic connectivity of fast-spiking interneurons in rat ventral striatum. Brain Res 1152:49-56.
Tse D, Langston RF, Kakeyama M, Bethus I, Spooner PA, Wood ER, Witter MP, Morris RG (2007) Schemas and memory consolidation. Science 316:76-82.

Tsodyks MV, Skaggs WE, Sejnowski TJ, McNaughton BL (1996) Population dynamics and theta rhythm phase precession of hippocampal place cell firing: a spiking neuron model. Hippocampus 6:271-280.

van der Meer MA, Redish AD (2009a) Covert expectation-of-reward in rat ventral striatum at decision points. Front Integr Neurosci 3:1.

van der Meer MA, Redish AD (2009b) Low and high gamma oscillations in rat ventral striatum have distinct relationships to behavior, reward, and spiking activity on a learned spatial decision task. Front Integr Neurosci 3:9.

van der Meer MA, Redish AD (2010) Expectancies in decision making, reinforcement learning, and ventral striatum. Front Neurosci 4:29-37.

van der Meer MA, Johnson A, Schmitzer-Torbert NC, Redish AD (2010a) Triple dissociation of information processing in dorsal striatum, ventral striatum, and hippocampus on a learned spatial decision task. Neuron 67:25-32.

van der Meer MA, Kalenscher T, Lansink CS, Pennartz CM, Berke JD, Redish $\mathrm{AD}$ (2010b) Integrating early results on ventral striatal gamma oscillations in the rat. Front Neurosci 4:28.

van Wingerden M, Vinck M, Lankelma J, Pennartz CM (2010) Theta-band phase locking of orbitofrontal neurons during reward expectancy. J Neurosci 30:7078-7087.

Voorn P, Vanderschuren LJ, Groenewegen HJ, Robbins TW, Pennartz CM (2004) Putting a spin on the dorsal-ventral divide of the striatum. Trends Neurosci 27:468-474.

Zar JH (1999) Biostatistical analysis, Ed 4. Upper Saddle River, NJ: Prentice-Hall. 